\title{
An Application of Context Middleware Based on Fuzzy Logic for Wireless Sensor Networks
}

\author{
Ye NING ${ }^{1,2}$, Ruchuan WANG ${ }^{2,3}$, Shouming MA ${ }^{2}$, Zhili WANG ${ }^{1}$ \\ ${ }^{1}$ Department of Information Science, Nanjing College for Population Program Management, Nanjing, China \\ ${ }^{2}$ Institute of Computer Science, Nanjing University of Post and Telecommunications, Nanjing, China \\ ${ }^{3}$ State Key Laboratory for Novel Software Technology, Nanjing University, Nanjing, China \\ Email: cathery163@163.com \\ Received May 25, 2009; revised July 22, 2009; accepted July 25, 2009
}

\begin{abstract}
The research of context-aware computing based on wireless sensor network (WSN) aims at intelligently connecting computers, users, and environment. So its application system should be flexibly adaptable to dynamic changes of context and application requirements and proactively provides the information satisfied with current context for users. The middleware can be very effective to provide the support runtime services for context-aware computing. In this paper we propose middleware architecture for context processing. This architecture is based on fuzzy logic control (FLC) system for context reasoning and sensor fusion. We propose a formal context representation model in which a user's context is described by a set of roles and relations correspond to a context space. A middleware prototype has been developed, which detect tourist' physical context and provide reminding. The experiments prove that the model and approach proposed are feasible.
\end{abstract}

Keywords: WSN, Context-Aware, Middleware, FLC, Pervasive Computing

\section{Introduction}

Context-aware has recently become a hot topic in the areas of pervasive computing, which was introduced by Schilit and Theimer [1]. With the development of wireless sensor networks (WSNs), the space and physical context information can be obtained by a large number of sensor nodes. How to intelligently integrating multiple sensors and sensor fusion is a crucial technology for context processing in WSNs.

To avoid increasing complexity and allow the users is to concentrate on his tasks, applications and services must be aware of their contexts and automatically adapt to their changing contexts-known as context-awareness.

In most situations, humans react opportunistically, switching among a set of possible goals, abandoning and adding new goals in response to events and opportunities. One of the most difficult challenges in designing context aware systems is to recognize and allow for such unpredictable behavior [2]. In this respect, middleware can be very effective to provide the support if they can reduce the effort required to develop distributed software and runtime services for applications with the abovementioned characteristics, in addition to providing the normal services, such as interoperability, location transparency, naming service, etc [3].

In this paper, we present the design and implementation of a middleware approach for context-awareness, and adopted fuzzy logic [4] as an intelligent reasoning method for selecting data dissemination protocols in the design of the decision mechanism.

The remainder of the paper continues as follows: in Section 2 discusses related work and main problem. In Section 3 we describe our formal context representation model and the structure of FLC system for Context processing. Section 4 describes an application we have developed for our middleware prototype. Finally, the paper ends with conclusion in Section 5.

\section{Relate Work and Problem Formulation}

We are not aware of any integrated middleware platform that aims to achieve all of the goals described above.

As early as in 2002, Huadong Wu etc. proposed a contextual information model and built a generalize-able sensor fusion software architecture that can support mapping sensors' raw output data into the contextual information hierarchy [5]. 
The Gaia project [6] developed at the University of Illinois is a distributed middleware infrastructure that provides support for ubiquitous computing.

The EasyLiving project [7] from Microsoft focuses on development of an architecture and technologies for intelligent environments.

Work at Illinois has developed the Universal Interoperable Core (UIC) which is a reflective middleware platform designed for handheld devices [8].

Recent research work has focused on middleware extensions for pervasive computing by standardizing on web services and service discover-oriented technology [9].

To sum up, it is important that middleware for context-aware application should be able to fulfill the following functionalities and objectives.

1) The middleware architecture should be modular and extensible.

2) The middleware should be based on a service-oriented architecture, in which each application and device is represented as a service entity.

Based on the above-mentioned study, we developed a middleware for pervasive computing, which adopted fuzzy logic as context processing method.

\section{Fuzzy Logic Based Context Processing}

\subsection{A Formal Context Representation Model}

We use a context space theory model shown in [10] for model fundamental nature of context and enable context and situation awareness for context processing. Our context model gives a common representation for context that all entities in the environment use of pervasive computing. Instead, it provides a common base on which various reasoning mechanisms can be specified to handle context.

\section{Definition 1:}

We define a attribute value $a_{i}$ as any type of data that is associated with Contextual information, include physical contexts, environmental contexts, informational contexts, personal contexts, social contexts, application contexts and system contexts [11].

\section{Definition 2:}

For a context state $C_{i}^{t}=\left(a_{1}^{t}, a_{2}^{t}, \cdots a_{N}^{t}\right)$, defined over a collection of $\mathrm{N}$ attribute-values, where each value $a_{i}^{t}$ corresponds to an attribute $a_{i}$ 's value at time t.

\section{Definition 3:}

Let $\mathrm{C}_{\text {space }}$ be a context space in an environment of pervasive computing, describes the application's current state in relation to chosen context. In our model, context spaces are represented as first-order calculus. The basic model has the form of predicate(subject, $\left.C_{a t t}\right)$, in which
- subject $\in S^{*}$ : set of the expression entity of context information, e.g. visitor, location, etc.

- predicate $\in V^{*}$ : set of predicate name, e.g. is located in, has status, etc.

$-\mathrm{C}_{\mathrm{att}} \in C^{*}$ : set of all values of context state in $S^{*}$, e.g. warm, cold, open, close, empty, etc.

For example, Location (Marry, laboratory) means Marry is located in the laboratory.

The basic context model can be extended to form a set of contexts by combining the predicate and Boolean algebra (union, intersection and complement).

For example,

BodyTemperature(Marry,38)^ Pluse(Marry,90) represents physical signs about Marry.

\subsection{Fuzzy Logic-Based Context Processing}

Fuzzy logic was proposed by Lotfi A. Zaheh in 1965 [12] to emulate the way that the human brain processes uncertainty, uncertainty, imprecision, and vagueness. Fuzzy logic is suitable for context management because it is capable of processing imprecise and unreliable information coming from pervasive computing, and it can describe a problem in a common sense format in which expert knowledge, instead of differential equations, can be applied [13].

For $\forall a_{i}, a_{i} \exists \mathrm{C}_{\text {space }}$, be an input is applied to a FLC system, the inference engine computes the output set corresponding to each rule. On analyzing the context processing of various potential services, we use singleton fuzzification and "IF-THEN" rules of form [14].

Rl: IF $a_{1}$ is $F_{1}^{l}$ and $a_{2}$ is $F_{2}^{l}$ and $\cdots$ and $a_{N}$ is $F_{N}^{l}$, THEN y is $G^{l}$.

Assuming singleton fuzzification, when an input $\mathrm{A}^{\prime}=\left\{a_{1}^{\prime}, \cdots, a_{N}^{\prime}\right\}$ is applied, the degree of firing corresponding to lth rule is computed as

$$
\mu_{F_{1}^{\prime}}\left(a_{1}^{\prime}\right) * \mu_{F_{2}^{l}}\left(a_{2}^{\prime}\right)^{*} \ldots * \mu_{F_{N}^{l}}\left(a_{N}^{\prime}\right)=\mathrm{T}_{i-1}^{N} \mu_{F_{i}^{l}}\left(a_{i}^{\prime}\right) \text { Where }
$$

* and $\mathrm{T}$ both indicate the chosen t-norm. In this paper, we focus on the height defuzzifier and used trapezoidal membership ship functions to represent low, high, very strong, very weak to represent moderate, medium, strong, and weak.

In this paper, we are primarily interested in developing middleware based on the structure of FLC mentioned above. We design a FLC system for travels services, which is one of component in our pervasive computing prototype Tourist Reminder.

The FLC system in this paper receives context information from sensor equipments as the inputs of the FLC and the fuzzification module converts inputs into fuzzy linguistic variable inputs. 
On analyzing the data requirements of travels services, four linguistic variables were defined, representing the physical sign of tourist. The membership functions of these input parameters of the fuzzy logic are illustrated in Figure 1.These member functions have been determined based on the simulation result. The labels in the fuzzy variables are presented as follows.

- Age $=\{$ infant, youth, midage, old $\}$;

- BodyTemp $=\{$ Normal $\}$;

- Pulse $=\{\mathrm{t} 1, \mathrm{t} 2, \mathrm{t} 3, \mathrm{t} 4\}$; //different intervals of normal pulse

- $\mathrm{R}=\{\mathrm{N}$ (Normal), L (Lower), H (Higher) $\} ; / /$ degree of reminding for physical signs

- Based on above fuzzy variables, we can define fuzzy IF-THEN rules such as follows.

1) If (Age is infant) and (Pulse is $t 1)$ then ( $R$ is $N$ )

2) If (Age is infant) and (Pulse is t2) then ( $R$ is $H$ )

3) If (Age is infant) and (Pulse is $t 3$ ) then ( $\mathrm{R}$ is $\mathrm{H}$ )

4) If (Age is infant) and (Pulse is $t 4)$ then ( $\mathrm{R}$ is $\mathrm{H}) \ldots . .$.

A sample fuzzy calculation at a value of context information point is described in Figure 2.

\section{Fuzzy Middleware Protoype Implementation}

We have evaluated the context processing mechanism that based on fuzzy logic system by developing a simple prototype application called Tourist Reminder. As Figure 3 shows, we use medical sensors (body temperature sensor, pulse sensor and blood oxygen sensor, etc) and GPS/RFID in detecting tourist's physical signs/location anywhere and anytime. All sensor data are transmitted by ZigBee wireless sensor nodes to the middleware running on PC or PDA for analyzing and providing reminding message to tourist.
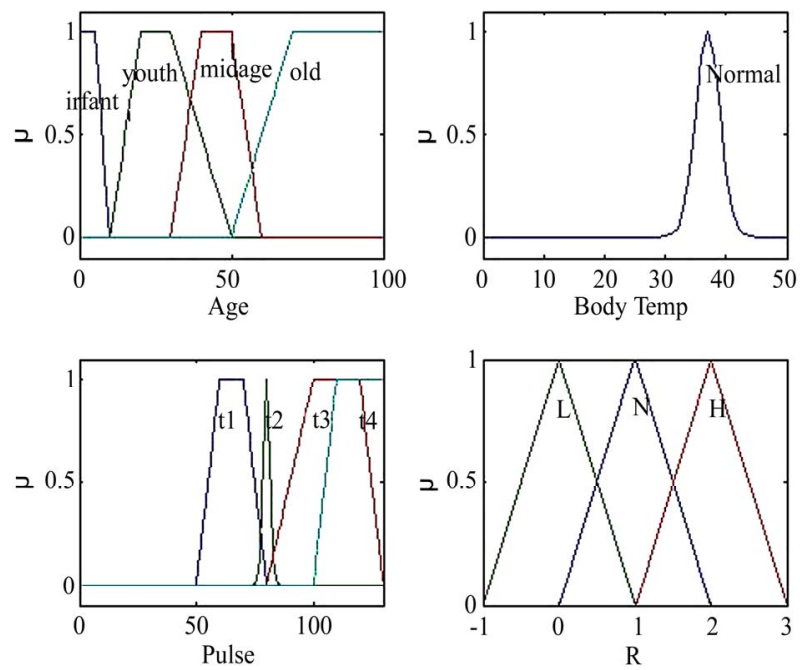

Figure 1. Membership functions for input context (physical signs).

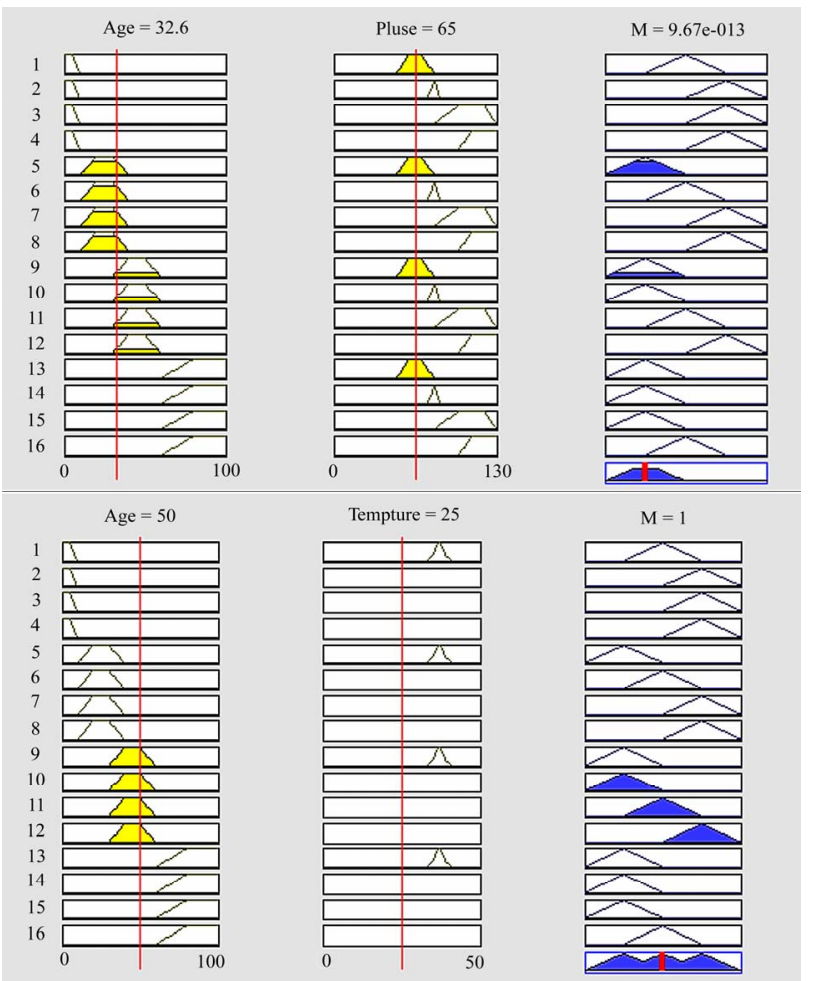

Figure 2. A sample fuzzy calculations.

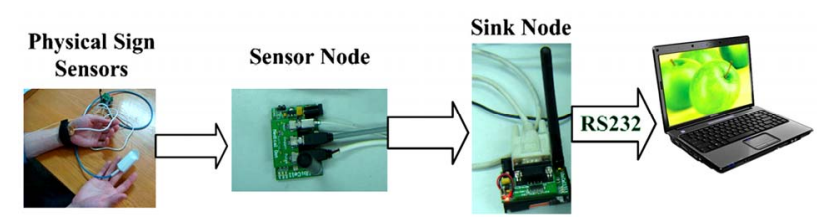

Figure 3. The process of prototype application.

\subsection{Middleware Architecture}

To create Tourist Reminder, we developed generic reference architecture applicable to pervasive computing space. As Figure 4 shows, the middleware contains separate physical, sensor platform, service, knowledge, context management, and application layers. Physical Layer: contains a variety of sensors and actuators which monitor and gather context information about the pervasive environment.

- Context Acquire Layer: integrates the sensors and actuators from the layer beneath and export their service representations to the layers above, which includes query processing component that can process filters and queries for sensor readings sent to it from the query processor in the service layer.

- Context service layer: contains the Open Services Gateway Initiative (OSGi) framework, which maintains leases of activated services. The layer provides the service discovery, composition, and invocation mechanisms for applications to locate and make use of particular 


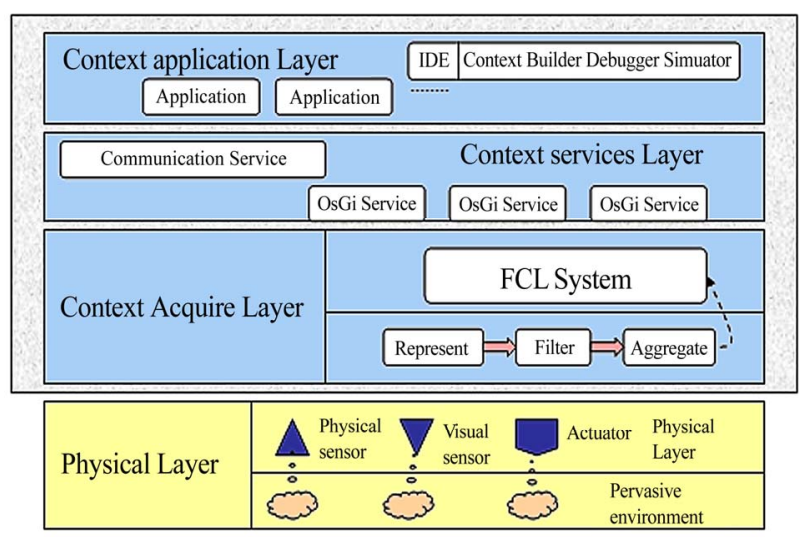

Figure 4.The architecture of middleware.

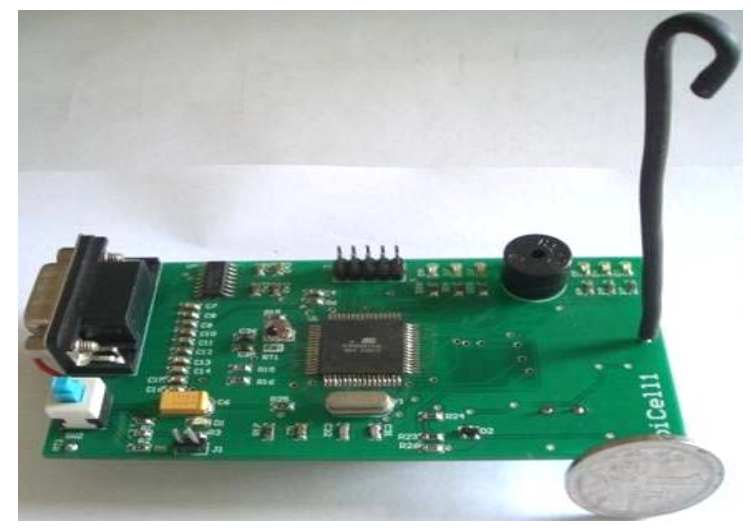

(a) UbiCell node

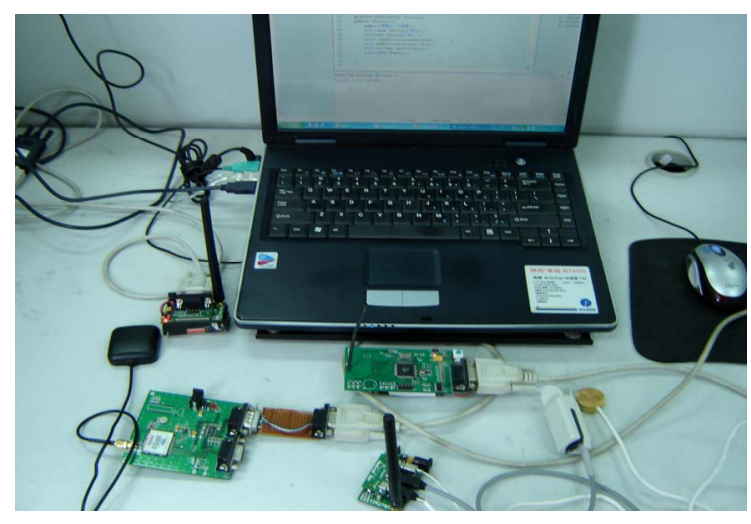

(b) physical sign sensors and PC

Figure 5. The hardware of Tourist Reminder.

sensors or actuators. It holds the registry of the software service representation of all sensors and actuators connected to the hardware nodes and FLC system as inference engine.

- Context application layer: sits at the top and consists of the execution environment that provides an API to access and control sensors, actuators, and other services. It contains a service authoring tool to enable rapid and efficient development and deployment of services and applications.

\subsection{Middleware Prototype Implement}

We develop sensor node called UbiCell[15], which ingrate models of GPS and physical sign sensors. The hardware of middleware prototype is composed of PC, UbiCell and sink nodes (Figure 5).

In this project, the develop platform of software was based on J2sdk1.4+Eclipse3.2.

As shows in Figure 6, the main function of Tourist Reminder include subscribed service, query service, message reminding, GIS location, etc.

- Client as the user of tourist service subscribes the services to Server (middleware) according to the ID about tourist.

- After sampling and aggregating the physical context about subscribed tourist, Server realizes reasoning based FLC system and returns the relevant messages of services (reminding/query).

Figure 7 is one of the capture images about sampling data of body temperature. As shows in Figure 7, the trend of data is stability.

\section{Conclusions}

Context-aware computing has been a key issue for pervasive computing based on WSN. In a pervasive computing environment, Services should be intelligent

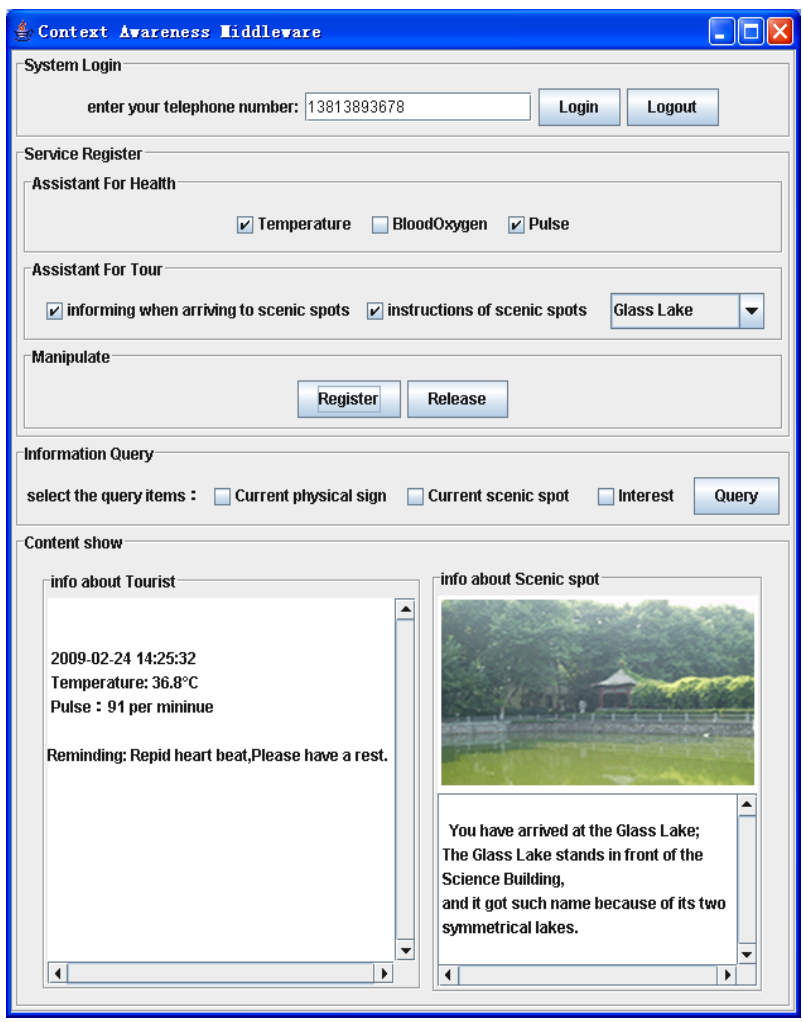

Figure 6. The interface of Tourist Reminder. 


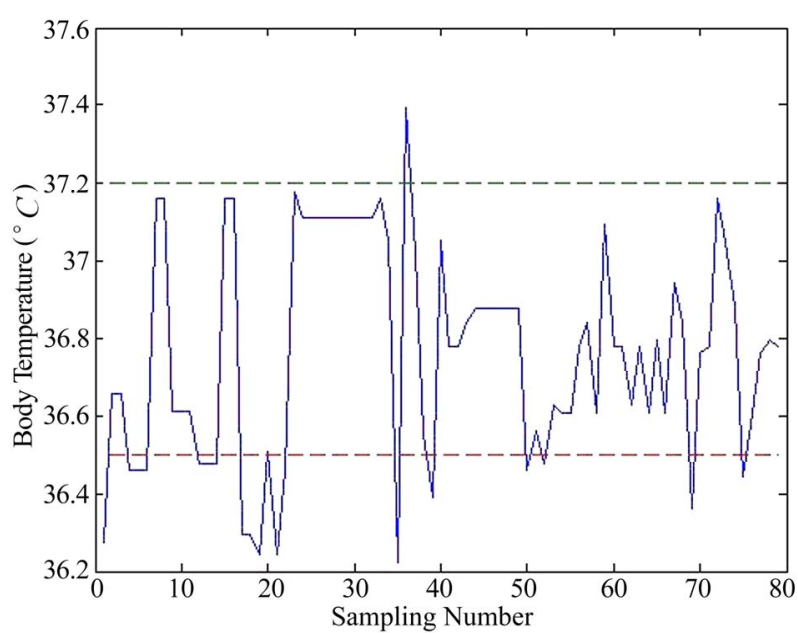

Figure 7. The capture image of sampling data.

enough to understand the real world. Our study in this paper demonstrates that fuzzy logic based middleware is feasible for facilitate context processing. A key feature of our model is the presence of FLC based context architecture.

The work of this paper is a part of our ongoing middleware prototype for pervasive computing which provides the reminding service to tourist. Now we furthering work are to apply in practice trade.

\section{Acknowledgements}

This work is supported by the National Natural Science Foundation of China (No. 60773041), Science Foundation of Jiangsu High School (No. 09KJB510020) and sponsored by Qing Lan Project.

\section{References}

[1] B. Schilit and M. Theimer, "Disseminating active map information to mobile hosts," IEEE Network, Vol. 8, No. 5, pp. 22-32, 1994.

[2] P. Dourish, "What we talk about when we talk about context,” Personal and Ubiquitous Computing, Vol. 8, No.
1, pp. 19-30, 2004.

[3] A. Helal, "Programming pervasive spaces," The Standards and Emerging Technologies Department, IEEE Pervasive Computing magazine, Sumi Helal, Dept. Editor, Vol. 4, No. 1, January-March 2005.

[4] H. J. Zimmermann, "Fuzzy sets theory and its applications," Second, revised edition, Kluwer Academic Publishers, 1991.

[5] H. Wu, M. Siegel, and S. Ablay, "Sensor fusion for context understanding," Instrumentation and Measurement Technology Conference, IMTC/2002, Proceedings of the 19th IEEE, Vol. 1, pp. 13-17, May 2002.

[6] M. Roman, C. K. H., R. Cerqueira, et al, "Gaia: A middleware infrastructure to enable active spaces,” IEEE Pervasive Computing, pp. 74-83, 2002.

[7] Microsoft Research, “Easy living,” 2009, http://research.microsoft.com/easyliving/.

[8] M. Roman, F. Kon, and R. H. Campbell, "Reflective middleware: From your desk to your hand,” IEEE DS Online (Special Issue on Reflective Middleware), 2001.

[9] S. Ou and K. Yang, "An effective offloading middleware for pervasive services on mobile devices,” Pervasive and Mobile Computing, Vol. 3, No. 4, pp. 362-385, 2007.

[10] A. Padovitz, et al, "An approach to data fusion for context awareness,” Fifth International Conference on Modelling and Using Context, CONTEXT'05, Paris, France, July 2005.

[11] M. Korkea-aho, “Context-aware applications survey,” 2009, http://www.hut.fi/ mkorkeaa/doc/context-aware.html.

[12] L. A. Zadeh, Fuzzy sets, Information and Control, pp. 338-353, 1965.

[13] M. Marin-Perianu, C. Lombriser, et al, "Distributed activity recognition with fuzzy enabled wireless sensor networks,” Technical Report TRCTIT-07-68, Enschede, September 2007.

[14] E. Hisdal, "The IF THEN ELSE statement and interval-valued fuzzy sets of higher type,” International Journal Man-Machine Studies, Vol. 35, pp. 385-455, 1981.

[15] Wireless Sensor Network Research Center of Nanjing University of Post and Telecommunications, "UbiCell product manuals,” 2009, http://www.wsns.net.cn. 